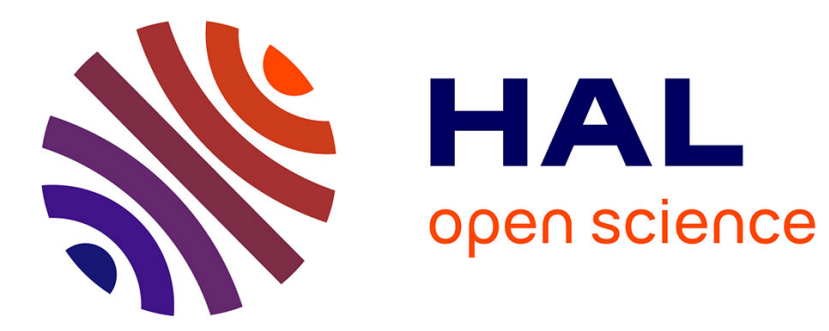

\title{
Punir les délinquants fiscaux en France et au Royaume-Uni : vers la convergence des modèles?
}

Alexis Spire, Katia Weidenfeld

\section{To cite this version:}

Alexis Spire, Katia Weidenfeld. Punir les délinquants fiscaux en France et au Royaume-Uni : vers la convergence des modèles?. Archives de politique criminelle, 2017. hal-02273402

\section{HAL Id: hal-02273402 \\ https://hal.science/hal-02273402}

Submitted on 28 Aug 2019

HAL is a multi-disciplinary open access archive for the deposit and dissemination of scientific research documents, whether they are published or not. The documents may come from teaching and research institutions in France or abroad, or from public or private research centers.
L'archive ouverte pluridisciplinaire HAL, est destinée au dépôt et à la diffusion de documents scientifiques de niveau recherche, publiés ou non, émanant des établissements d'enseignement et de recherche français ou étrangers, des laboratoires publics ou privés. 


\title{
Punir les délinquants fiscaux en France et au Royaume-Uni : vers la convergence des modèles $^{1}$ ?
}

\author{
Alexis Spire (CNRS -PSL) \\ Katia Weidenfeld (Ecole Nationale des Chartes -PSL)
}

\section{Introduction}

Dans la lignée des travaux d'Edwin Sutherland ${ }^{2}$, quelques études ont envisagé le traitement de la fraude fiscale sous l'angle de la tendance des institutions à traiter avec mansuétude ce type de délinquance en col blanc. Des analyses convergentes ont ainsi été proposées de part et d'autre de la Manche dans les années 1980. En France, Pierre Lascoumes a mis en lumière les filtres successifs qui s'exercent avant les poursuites ${ }^{3}$ : en abordant les manquements à l'impôt comme des erreurs plutôt que comme des fraudes, l'administration contribue à leur invisibilité. Pour la Grande Bretagne, Dee Cook a également montré comment les fonctionnements institutionnels conduisaient à un traitement de la fraude fiscale beaucoup plus conciliant que celui de la fraude sociale ${ }^{4}$. Mais depuis ces travaux pionniers, l'essentiel des recherches académiques des concentre sur les mécanismes qui incitent les contribuables à frauder, ou à respecter la loi ${ }^{5}$.

La fin des années 2000 a pourtant modifié le contexte d'indifférence qui prévalait jusqu'alors. La révélation des évasions fiscales commises par des ressortissants de nombreux pays européens vers des banques luxembourgeoises a joué le rôle de déclencheur. Au Royaume-Uni comme en France, en particulier, les politiques publiques accordent une importance renouvelée à la fraude fiscale et remettent sur le devant de la scène les instruments de pénalisation. A partir de la fin 2009, les gouvernements qui se succèdent au 10 Downing Street incitent l'administration fiscale à accroître le nombre de poursuites pénales contre des fraudeurs fiscaux. La décision de charger le Crown Prosecution Service de ces poursuites

\footnotetext{
${ }^{1}$ Cet article reprend, synthétise et prolonge des éléments développés dans l'étude Des erreurs ou des fraudes? Le sens des sanctions en matière fiscale, menée pour le compte de la Mission Droit et Justice.

${ }^{2}$ Edwin SUTHERLAND, White collar crime, New York: Holt, Rinehart \& Winston, 1949.

${ }^{3}$ Pierre LASCOUMES, «Sanction des fautes ou gestion des illégalismes : l'hétérogénéisation du droit pénal. Un exemple, la répression fiscale », Revue interdisciplinaire d'études juridiques, 10, 1983, p. 125-156.

${ }^{4}$ Dee COOK, Rich Law, Poor Law. Differential Response to Tax and Supplementary Benefit Fraud, Open University Press, 1989.

${ }^{5}$ Pour une synthèse, voir Jeffrey A. ROTH, John T SCHOLZ (ed.), Taxpayer Compliance. An Agenda for Research, Philadelphia, University of Pennsylvania Press, 1979 p. 71 sq.
} 
symbolise la volonté de rapprocher le traitement de ce délit de la délinquance conventionnelle. Presque simultanément en France, une loi crée une nouvelle procédure de répression de la fraude fiscale, qui se superpose à l'ancienne. Alors que, classiquement, l'enquête judiciaire n'intervient qu'à l'issue du contrôle fiscal, il est désormais permis à l'administration fiscale de transmettre directement à l'institution judiciaire, avant d'avoir achevé son propre travail, les dossiers les plus significatifs (une centaine par an). Le ministre du Budget conserve l'initiative des poursuites pour fraude fiscale dans tous les cas, à travers ce que les médias ont dénommé le « verrou de Bercy ». Mais l'utilisation de la qualification de «blanchiment de fraude fiscale » (quelques cas $\operatorname{par} \mathrm{an}^{6}$ ) permet aux procureurs de passer outre.

Des deux côtés de la Manche, la lutte contre la fraude fiscale est devenue un sujet de communication politique. En témoignent le discours du directeur du Crown Prosecution Service de janvier $2013^{7}$ ou les deux nouvelles lois adoptées en France en décembre 2013, suite à «l'affaire Cahuzac ». Les déclarations d'intransigeance se multiplient, revendiquant à la fois de couvrir l'ensemble des délits fiscaux - des plus simples aux plus sophistiqués- et de punir sévèrement les délinquants pour faire des exemples.

L'objet de cet article est d'étudier la mise en pratique effective de cette nouvelle politique pénale en France, en utilisant le cas britannique comme cadre de comparaison. Contrairement à la plupart des études théoriques et empiriques relatives à la fraude fiscale ${ }^{8}$, l'enjeu n'est pas d'évaluer l'efficience des instruments mis en place pour convaincre les contribuables de respecter leurs obligations fiscales. Notre propos est de montrer qu'en dépit des déclarations de fermeté, les agents de l'Etat restent prisonniers de règles, de pratiques et de représentations institutionnelles qui conduisent, en pratique, à une certaine tolérance à l'égard de la fraude fiscale.

Cette tolérance se traduit, dans les deux pays étudiés, par le faible nombre de poursuites pénales pour fraude fiscale. Le tri n'est cependant pas uniforme dans les deux pays : les mécanismes de sélection en amont permettent aux puissants d'échapper aux

\footnotetext{
${ }^{6}$ Aucune statistique ne paraît disponible sur ce point. Mais cette qualification semble surtout utilisée, à titre autonome, par le Parquet national financier.

${ }^{7} \mathrm{http}: / /$ www.cps.gov.uk/news/articles/prosecuting tax evasion/ (en ligne le 19 avril 2017).

8 Steven KLEPPER, Daniel NAGIN, "The Anatomy of Tax Evasion", Journal of Law, Economics, \& Organization, vol. 5, n 1, 1989, pp. 1-24 ; John BRAITHWAITE, Crime, Shame and Reintegration, Cambridge, Cambridge University Press, 1989 ; Valerie BRAITHWAITE, "Responsive Regulation and Taxation : Introduction", Law and Society, vol. 29 (1), 2007, pp. 3-10 ; Kristina MURPHY, "Enforcing Tax Compliance : To Punish or Persuade ?”, Economic analysis \& Policy, vol. 38, n 1, 2008, pp. 113-135. Et pour une critique, Michael LEVI, "Serious tax fraud and noncompliance : A review of evidence on the differential impact of criminal and noncriminal proceedings", Criminology \& Public Policy, 2010, vol. 9, issue 3, pp. 493-513.
} 
procédures pénales tandis que les petits indépendants y sont plus souvent exposés. Si cette gestion différentielle des illégalismes ${ }^{9}$ qui bénéficie aux grandes entreprises et aux contribuables fortunés pouvait sembler plus marquée en France qu'au Royaume-Uni, les évolutions des dernières années font, dans une certaine mesure, converger les deux modèles.

Les différences entre les deux pays restent en revanche sensibles concernant les sanctions. Bien que les réformes récentes en France aient relevé le niveau des peines susceptibles d'être prononcées contre les fraudeurs, celles-ci conservent un caractère largement virtuel. En comparaison, la répression des fraudeurs reconnus coupables est beaucoup plus sévère au Royaume-Uni. Cette différence traduit des conceptions distinctes du rôle de la sanction pénale dans la procédure fiscale. Dans les deux pays, la répression est censée endosser une fonction préventive (décourager les contribuables potentiellement déviants). Mais celle-ci n'est confortée par une fonction rétributive (imposer les stigma du crime) qu'au Royaume-Uni. En France, la sanction pénale demeure essentiellement un moyen de renforcer les pouvoirs du fisc pour recouvrer les impôts fraudés.

\section{Les matériaux utilisés}

L'étude conduite en France comporte un premier volet qualitatif qui consiste en une série d'entretiens menés avec les différents acteurs de la répression (agents de l'administration fiscale, policiers, procureurs, avocats et juges) et en l'observation d'audiences. Elle s'appuie également sur un échantillon représentatif de 570 cas de fraude fiscale soumis à l'institution judiciaire et terminés par un jugement ou arrêt rendu en 2011. Nous avons ainsi pu déterminer les propriétés sociales (notamment âge, nationalité, professions) des contribuables mis en cause et les sanctions qui leur étaient appliquées.

Pour développer une comparaison avec la Grande-Bretagne, des entretiens avec les différents acteurs de la lutte contre la fraude fiscale (agents d'HMRC et du CPS, barristers) ont été réalisés et plusieurs procès observés. Les informations statistiques publiées ont été complétées par des Freedom of information Requests, mais malheureusement aucun élément sur les propriétés sociales des contribuables poursuivis ne nous a été communiqué.

\section{Des machines à trier les manquements fiscaux}

Bien que les deux pays aient des règles de sélection des délinquants fiscaux poursuivis pénalement très différentes, leurs fonctionnements produisent, en pratique, des effets proches.

\footnotetext{
${ }^{9}$ Michel FOUCAULT, Surveiller et Punir. Naissance de la prison, Paris, Gallimard, 1975.
} 
Comme tous les crimes en col blanc, la fraude fiscale est très faiblement pénalisée ${ }^{10}$. Sur les 50.000 contrôles approfondis réalisés chaque année en France, environ 16.000 sont considérés par les inspecteurs comme des fraudes délibérées. Toutes pourraient être poursuivies pénalement, mais l'administration s'en tient généralement à des amendes et ne traduit devant les juridictions pénales qu'environ 1000 contribuables par an. Au Royaume Uni, la sélection est également drastique : le nombre de poursuites pour fraude fiscales reste légèrement inférieur à 900 depuis 2013-2014.

En matière de fraude fiscale, comme pour d'autres délits en col blanc ${ }^{11}$, plusieurs institutions jouent le rôle de filtres. En France, l'essentiel de la sélection des contribuables poursuivis est effectué par les agents de l'administration fiscale : l'inspecteur des impôts et son chef de brigade font des propositions à la direction départementale qui sélectionne certains cas puis les soumet au niveau central. Il faut ensuite qu'une «Commission des infractions fiscales » composée de hauts-fonctionnaires donne son autorisation. La plainte est ensuite transmise au procureur de la République (autorité de poursuite insérée dans l'institution judiciaire) qui théoriquement peut refuser de poursuivre certains délinquants, mais qui, en pratique, n'exerce qu'exceptionnellement ce pouvoir. Au Royaume Uni, le fisc ne dispose pas, en droit, du monopole de l'action contre les auteurs d'évasion fiscale. Mais, en fait, le Crown Prosecution Service ou même les agences spécialisées, tel le Serious Fraud Office, ne poursuivent pas les cas de fraude fiscale sans associer Her Majesty Revenue and Customs (HMRC). Le Crown Prosecution Service -dont les moyens ne cessent de décroîtreest plus actif que le Parquet français dans le tri des infractions poursuivies : il refuse environ $15 \%$ des dossiers venant de $H M R C$.

En dépit de ces différences institutionnelles, le choix de poursuivre pénalement tel fraudeur du fisc plutôt que tel autre, revient ainsi principalement, en France comme en Grande Bretagne, à l'administration fiscale. Des deux côtés de la Manche, cette activité de tri conduit à diriger l'essentiel des poursuites contre les contribuables qui, même après avoir été alertés des soupçons pesant sur eux, refusent de « coopérer ».

${ }^{10}$ David WEISBURD, Stanton WHEELER, Elin WARING, Nancy BODE, Crimes of the Middle Classes. White-Collar Offenders in the Federal Courts, New Haven, Yale University Press, 1991.

${ }^{11}$ David J. EITLE, "Regulatory justice: A re-examination of the influence of class position on the punishment of white-collar crime”, Justice Quarterly, 17 (4), 2000, pp. 809-839. 


\section{La gestion différentielle des illégalismes fiscaux}

Dans les deux pays, l'administration fiscale n'oriente vers le pénal que les contribuables les plus récalcitrants. Cette philosophie rend possible une gestion différentielle des illégalismes fiscaux qui profite aux plus puissants.

Celle-ci profite d'abord aux grandes entreprises. Dans notre échantillon de 570 jugements de fraude fiscale, aucune plainte pénale n'a été déposée par la DVNI, le service chargé du contrôle des plus grandes entreprises, alors qu'il notifie plus du tiers des rectifications $^{12}$. Plusieurs facteurs concourent à cette faible pénalisation. Tout d'abord, les grandes entreprises, accompagnées par des professionnels du droit, parviennent à déplacer la frontière entre le légal et l'illégal ${ }^{13}$. A la suite du séisme provoqué par l'affaire Enron, les Etats occidentaux ont accentué leur intérêt pour l'optimisation agressive. La France (avec la théorie juridique de la fraude à la loi) comme le Royaume Uni ${ }^{14}$ ont renforcé les instruments permettant de combattre l'utilisation par ces contribuables de la lettre de la loi afin de mettre en échec son esprit. Mais même débusquées, les fraudes à la loi continuent à bénéficier d'une apparence légale. Les techniques sont parfois remises en cause au civil mais leurs auteurs échappent généralement aux poursuites criminelles ${ }^{15}$ : le principe d'interprétation stricte de la loi pénale rend incertain le succès de procédures répressives à leur encontre.

Les entreprises, et notamment les plus grandes, continuent en outre à jouir d'un crédit de réputation, en raison des emplois qu'elles créent ou des produits de consommation qu'elles distribuent; il y a là un frein à l'engagement de poursuites pénales qui pourraient menacer leur survie ${ }^{16}$. Cette représentation explique sans doute que les actions pénales engagées, à la suite des réformes de 2013, contre des grandes entreprises ont essentiellement visé le secteur discrédité de la banque et principalement contre des sociétés étrangères (en France, la filière suisse d'HSBC et la banque suisse UBS AG).

Les plus grandes entreprises ne sont d'ailleurs pas les seules à échapper massivement à toute poursuite pénale. En France, les contribuables les mieux dotés, en capital économique ou social, en sont également largement exempts. Le service chargé de leur contrôle -dont le

${ }^{12}$ Cour des comptes 2012

${ }^{13}$ Carla NAGELS, «Les grandes entreprises et les instances de lutte contre la fraude sociale : le jeu du chat et de la souris », Champ pénal, 10, 2013.

${ }^{14}$ General Anti-Abuse Rule 2013.

15 Doreen MC BARNETT, “After Enron will still 'Whiter than white collar crime' still wash ?”, British Journal of Criminology, 46, 2006, pp. 1091-1109.

${ }^{16}$ David O. FRIEDRICH, Trusted Criminals: White Collar Crime in Contemporary Society, Belmont, CA: Wadsworth, 2009. Cet objectif a été clairement énoncé lors de la création de la convention judiciaire d'intérêt public par la loi Sapin II : les entreprises ont obtenu d'échapper aux poursuites en payant une somme d'argent, de façon à éviter que l'accès aux marchés publics ne leur soit fermé. 
portefeuille est sensiblement plus large que celui de son homologue britannique ${ }^{17}$ - est à l'origine de seulement environ $2 \%$ des plaintes. Les membres des professions libérales qui fraudent l'impôt sont également très préservés de toute poursuite pénale dans l'Hexagone. Dans notre échantillon de jugements, les avocats, experts-comptables, architectes et médecins représentent moins de $6 \%$ des personnes poursuivies. Les raisons de cette faible criminalisation des cols blancs sont en partie similaires à celles relatives aux grandes entreprises. Entourés d'avocats habiles à donner à leurs fraudes l'apparence d'erreurs ${ }^{18}$, les «grandes fortunes » discutent et négocient abondamment avec les services fiscaux. S'ils ne bénéficient pas d'une impunité totale, ils saisissent plus facilement les options leur permettant d'écoper de sanctions administratives et d'échapper aux sanctions pénales ${ }^{19}$. En outre, leurs fraudes concernent souvent les impôts patrimoniaux, comme les droits de succession ou l'impôt de solidarité sur la fortune, qui sont moins bien acceptés que d'autres prélèvements ${ }^{20}$.

Cette gestion différentielle des illégalismes s'explique également par la persistance d'un objectif primordial pour les inspecteurs des impôts : faire rentrer, à court terme, de l'argent dans les caisses de l'Etat. La volonté affichée par les hauts fonctionnaires français de cibler davantage les fraudes patrimoniales se heurte ainsi à un but de rentabilité qui l'emporte systématiquement sur le souci d'aboutir à des sanctions criminelles. De fait, les inspecteurs spécialisés dans le contrôle des patrimoines ont tendance à considérer que si le contribuable accepte de s'acquitter au moins d'une partie des rectifications, l'engagement d'une procédure pénale est superflu. L'objectif de recouvrement des impôts fraudés auquel sont soumis les agents des impôts explique également la rareté des poursuites dirigées contre les intermédiaires -avocats ou experts-comptables notamment- qui imaginent la fraude sans en bénéficier directement : l'administration fiscale qui est à l'initiative des poursuites s'intéresse prioritairement aux contribuables qui sont nommément bénéficiaires de la fraude et assez peu aux professionnels qui l'ont rendue possible.

${ }^{17}$ La High Net Worth Unit se concentre sur les 5800 contribuables dont le patrimoine dépasse 20 millions de livres alors que la Direction Nationale des Vérifications de Situations Fiscales contrôle les particuliers dont le revenu est supérieur à 770.000 euros, ou le patrimoine supérieur à 6,9 millions d'euros ; s'y ajoutent les contribuables à la notoriété avérée et ceux qui font l'objet d'une procédure judiciaire, soit au total environ 150.000 personnes.

${ }^{18}$ Kenneth MANN, Defending white collar crime: A portrait of attorney at work, New Haven: Yale University Press, 1985.

${ }^{19}$ Susan P. SHAPIRO, "Collaring the crime, not the criminal: Reconsidering the concept of white-collar crime", American Sociological Review, 1990, p. 362

${ }^{20}$ Jens BECKERT, "The longue durée of Inheritance Law", European Journal of Sociology, 48(01), 2007, pp. 79-120. 
Ce dernier frein à la pénalisation des fraudes des plus fortunés joue moins fortement outre-Manche. Le choix d'orienter un dossier vers la voie pénale y est en effet effectué de manière plus précoce : il intervient en amont ou au cours du contrôle fiscal. Or, les agents chargés de l'enquête criminelle au sein d'HMRC ont des objectifs de nature clairement pénale (recherche d'une conviction et d'une condamnation), qui ne viennent pas interférer avec les objectifs budgétaires poursuivis par l'administration fiscale dans son ensemble. En GrandeBretagne, le fait que le contribuable paye les impôts dus n'influe donc en général pas sur la décision de poursuivre la procédure pénale.

Par ailleurs, l'administration fiscale britannique assume de cibler particulièrement certaines professions, notamment les professionnels du droit. A leur encontre, la politique affichée est d'engager des poursuites dès lors que la fraude est démontrée et souvent sans leur laisser la chance d'une amnistie, même si le montant en jeu est relativement limité. Quelques juristes ont ainsi été inquiétés, non seulement pour leur propre fraude mais aussi pour celles conseillées à leurs clients. C'est le cas par exemple d'un expert fiscal à l'origine de la vente à des clients aisés de montages frauduleux au crédit d'impôt ${ }^{21}$, ou d'un brillant diplômé d'Oxford accusé d'avoir siphonné une niche fiscale environnementale 22 .

Les réformes du début des années 2010 ont vocation à rapprocher les pratiques françaises du modèle anglais. Elles permettent de faire démarrer la procédure pénale avant la fin du contrôle fiscal et de replacer l'objectif répressif au centre de celle-ci. Le renvoi devant un tribunal correctionnel des anciens ministres Cahuzac et Thévenoud, bien qu'ils se soient acquittés de l'ensemble des impôts et amendes dus, en atteste. De même, la condamnation prononcée à l'encontre de l'avocat d'Arlette Ricci ou les poursuites pour complicité de fraude fiscale contre un éditeur de logiciels effaçant certaines recettes dans la comptabilité des officines pharmaceutiques ${ }^{23}$ sont très emblématiques d'une volonté nouvelle d'atteindre les instigateurs. Néanmoins, ces évolutions ont un effet marginal. Ainsi, le Parquet national financier, qui joue un rôle moteur dans cet effort pour traquer la délinquance des puissants, ne traite que quelques dizaines de fraudes fiscales par an. Au total, cette nouvelle politique pénale a des résultats limités ${ }^{24}$.

\footnotetext{
${ }^{21}$ Robert Marshall Faichney et David Richard Perrin, Blackfriars Crown Court, 7 février 2012.

${ }^{22}$ Jonathan Anwyl, Southwark Crown Court, 12 mars 2017.

${ }^{23}$ Tribunal correctionnel de Nîmes, 17 mars 2017.

${ }^{24}$ En 2014, seules trois plaintes concernaient des impôts autres que la TVA, l'impôt sur les sociétés et l'impôt sur le revenu, cf. Commission des infractions fiscales, Rapport annuel, 2015.
} 


\section{Les petits entrepreneurs, clients habituels des tribunaux}

Dans les deux pays, le cantonnement des poursuites pénales aux contribuables qui refusent le dialogue et la négociation avec le fisc se traduit par la place importante de fraudes sans complexité technique.

Dans près d'un tiers des cas en France, il s'agit simplement d'une absence de déclaration $^{25}$. C'est le cœur du délit reproché aux gérants d'entreprise du bâtiment et des travaux publics qui représentent le quart des plaintes fiscales françaises. Pour la plupart, ces entrepreneurs ne répondent pas aux courriers du fisc, ne présentent aucune comptabilité lors du contrôle et sont, d'ailleurs, souvent jugés par contumace. Notre base statistique révèle par ailleurs la surreprésentation des étrangers, parmi ces chefs d'entreprise : $46 \%$ des entrepreneurs du bâtiment poursuivis pénalement pour fraude fiscale sont de nationalité étrangère et les deux tiers sont nés hors de France (alors que les personnes résidant en France nées hors du territoire représentent seulement $11,5 \%$ de la population). De manière plus générale, près du quart des personnes poursuivies pour fraude fiscale ne sont pas françaises, alors que la population étrangère ne représente que $6 \%$ de la population résidant en France. La surreprésentation des étrangers dans la répression de la fraude fiscale ( $25 \%$ des prévenus) est beaucoup plus élevée que dans la délinquance générale (environ 12\%) et même que dans la délinquance économique et financière (environ 14\%) (Casier judiciaire national 2010-2013). Les Turcs semblent être les contribuables les plus souvent ciblés : dans notre échantillon, ils sont à eux seuls visés par $10 \%$ des poursuites alors qu'ils ne représentent que $0,3 \%$ de la population française. De tels écarts statistiques soulèvent la question d'une éventuelle discrimination émanant des services chargés de réprimer la fraude fiscale. En pratique, il est très difficile de la prouver car le critère de la nationalité ne peut être considéré indépendamment du comportement du contribuable face à l'impôt et aux injonctions des vérificateurs. Tout comme dans le cas des jugements de droit commun plus sévères contre certaines minorités ${ }^{26}$, une part importante de la sur-pénalisation des étrangers ayant fraudé l'impôt s'explique par la nature et les circonstances des infractions : les Turcs mis en cause sont par exemple très souvent gérants (réels ou factices) de petites entreprises du bâtiment qui ne déposent pas de déclarations d'impôts et qui ne répondent à aucune sollicitation de l'administration.

\footnotetext{
${ }^{25}$ Voies et Moyens, Annexe au projet de loi et finances pour 2014, t. I : les recettes.

${ }^{26}$ Roger HOOD, Race and sentencing. A study in the crown court. Report for the commission for racial equality, Oxford : Clarendon Press, 1992 ; Fabien JOBARD, Sophie NEVANEN, «La couleur du jugement », Revue française de sociologie, 48(2), 2007, pp. 243-272.
} 
Ce biais de sélection est doublé par la présence fréquente, parmi les poursuivis, de personnes qui n'assument la direction de l'entreprise que sur le papier. Les magistrats interviewés déplorent d'avoir régulièrement en face d'eux des gens qui ont été démarchés pour ouvrir un compte au nom de la société et signer quelques documents, en échange de menus avantages; dans notre échantillon de jugements, ces cas représentent $10 \%$ des personnes poursuivies. Cette forme de sous-traitance de l'illégalité, très courante dans le bâtiment ${ }^{27}$, est aussi très répandue pour couvrir des situations de fraude fiscale.

En Grande Bretagne, les fraudeurs de faible envergure représentent également une part importante des cas déférés devant les tribunaux criminels. Suite au Comprehensive Spending Review 2010, l'augmentation des moyens dédiés aux investigations criminelles devait permettre de détecter un vaste éventail de fraudes incluant non seulement les montages complexes vendus par des conseillers fiscaux sans scrupule mais aussi les évasions fiscales peu sophistiquées et de moindre ampleur ${ }^{28}$. Les agents d'HM Revenue and Customs que nous avons interrogés reconnaissent consacrer au moins la moitié de leur temps à des affaires simples, concernant des contribuables qui s'abstiennent simplement de déclarer leurs impôts ou leur chiffre d'affaires. La couverture de l'ensemble du spectre de la fraude fiscale, y compris la plus triviale, est présentée comme un élément de dissuasion. L'objectif revendiqué des brigades spécialisées dans le contrôle des activités réputées être exposées à la fraude comme les restaurants, les fast-foods, le commerce automobile, l'immobilier, les sociétés de sécurité, les concierges de Londres...- est de faire preuve de sévérité. Les affaires qu'ils présentent à la Serious Evasion Team ont ainsi, selon les agents interviewés, de fortes probabilités d'être retenues pour la voie pénale. C'est le cas, par exemple, de deux restaurateurs, à la double nationalité anglaise et bangladaise, poursuivis pour n'avoir souscrit aucune déclaration d'impôt pendant les cinq dernières années ${ }^{29}$. Les campagnes d'amnistie visant les plombiers ou les professions du bâtiment ont des effets analogues : ceux qui n'ont pas saisi l'opportunité de mettre leurs affaires en règle ont été ciblés pour des poursuites pénales. Les agents d'HMRC sélectionnent les cas qui ont de bonnes probabilités de se conclure par une reconnaissance de culpabilité (qui intervient, selon les agents interviewés,

${ }^{27}$ Sebastien CHAUVIN, Nicolas JOUNIN, « L’externalisation des illégalités. Ethnographies des usages du travail 'temporaire' à Paris et à Chicago », Laurence FONTAINE, Florence WEBER (dir.), Les paradoxes de l'économie informelle. À qui profitent les règles ?, Paris, Karthala, 2011, pp. 113-138.

${ }^{28} \mathrm{HMIC}$, An Inspection of Her Majesty's Revenue and Customs performance in addressing the recovery of crime from tax and duty evasion and benefit fraud, Revisit 2013, p. 11.

${ }^{29}$ Southwark Crown Court, 1er juillet 2014. 
dans environ $60 \%$ des cas) - ce qui permet d'éviter un procès onéreux. Ce souci d'efficience conduit à pénaliser des contribuables souvent relativement démunis.

Ainsi, en France comme en Grande Bretagne, les prévenus qui doivent répondre de fraude fiscale devant le juge pénal sont drastiquement sélectionnés et ne représentent qu'une infime partie des contribuables ayant délibérément esquivé l'impôt. Si les modalités institutionnelles de tri sont différentes des deux côtés de la Manche, les deux pays pratiquent, à leur manière, une gestion différentielle des illégalismes fiscaux : les gérants de petites entreprises sont très présents devant les tribunaux alors que les dirigeants des grands groupes sont beaucoup plus rarement confrontés au juge pénal. En revanche, l'issue des procès est très différente d'un pays à l'autre.

\section{Des armes pour punir les fraudeurs?}

En France comme en Grande-Bretagne, les agents chargés de sélectionner les fraudes à poursuivre n'en conservent qu'une infime proportion et ciblent les dossiers dans lesquels les preuves sont les plus solides, ce qui induit une condamnation presque systématique $(90 \%$ des deux côtés de la Manche ${ }^{30}$ ) pour les quelques centaines de contribuables poursuivis.

Dans les deux pays, les peines maximales dont peuvent en principe écoper les délinquants sont relativement élevées : dix ans pour un grand nombre de qualifications au Royaume-Uni et cinq ou sept ans en France. En pratique, dans les deux pays, les condamnations effectivement prononcées sont loin des maxima légaux. Mais le décalage est beaucoup plus marqué en France. Les sentences britanniques continuent à donner une large place aux peines visibles, attirant la honte sur le contribuable. En France, les peines ont au contraire un caractère largement virtuel et dans bien des cas, les juges cherchent plutôt à éviter toute stigmatisation. Tout se passe comme si les poursuites pénales étaient avant tout un instrument accordé à l'administration fiscale pour améliorer le recouvrement des impôts fraudés.

\section{L'enfermement}

En France, malgré une légère accentuation du recours à cette sanction depuis 2010, la prison ferme n'est prononcée que dans $10 \%$ des condamnations pour fraude fiscale ${ }^{31}$. Dans notre échantillon de 570 jugements, les rares cas dans lesquels un délinquant fiscal sans casier

\footnotetext{
${ }^{30}$ https://www.gov.uk/government/news/beating-the-tax-cheats-hmrcs-criminal-case-highlights-of-2016 (version en ligne le 20 avril 2017).

${ }^{31}$ Voies et Moyens, Annexe au projet de loi et finances pour 2014, t. I : les recettes.
} 
judiciaire est condamné à une peine de prison ferme sont de deux types : soit une connexion existe entre la fraude fiscale et une délinquance conventionnelle (notamment trafic d'armes ou de stupéfiants), soit le contribuable refuse effrontément de faire amende honorable devant le tribunal. Et dans ce cas, les condamnations prononcées renvoient à de courtes durées d'emprisonnement. Or en-deçà de deux ans, la peine est «aménageable », c'est-à-dire que le condamné qui présente suffisamment de garanties d'insertion peut demander à exécuter sa peine sans incarcération (par exemple sous surveillance électronique). En définitive, alors que 5000 délinquants en col blanc sont derrière les barreaux en France ${ }^{32}$, aucun ne paraît y être pour le seul délit de fraude fiscale. Même dans les deux procès menés sous la houlette du Parquet national financier où des peines « exemplaires » de prison ferme ont été prononcées contre des primo-délinquants, Arlette Ricci et Jérôme Cahuzac, les juges ne les ont pas assorties d'un mandat de dépôt. En appel, la peine de prison prononcée contre l'héritière de la couturière a d'ailleurs été entièrement assortie du sursis.

Dans $80 \%$ des condamnations pour fraude fiscale, c'est ainsi une peine de prison avec sursis, d'une durée moyenne de neuf mois, qui est prononcée ${ }^{33}$. Or dans ce domaine, le sursis ne peut pas véritablement jouer la fonction d'avertissement puisque les jugements interviennent au moins cinq ans après les faits. Même pour un fraudeur fiscal endurci, la probabilité d'une nouvelle condamnation révoquant le sursis est faible...

Les magistrats interrogés justifient l'absence de condamnation à des peines de prison ferme de deux manières. Ils relèvent, d'une part, la bonne insertion sociale des délinquants fiscaux et reconnaissent être réticents à mettre à l'écart de la société des personnes perçues comme ayant une activité productive (en activité, voire employeur). Ils reprennent ainsi pleinement à leur compte la thèse selon laquelle la fonction de l'incarcération dans la société contemporaine est bien davantage de réguler la pauvreté que de punir le crime ${ }^{34}$. Les magistrats expliquent, d'autre part, le fréquent recours au sursis par le fait que les prévenus qu'ils ont à juger sont souvent des hommes de paille, qui, s'ils ont prêté mainforte à la fraude, ne l'ont pas imaginé et n'en ont que peu profité. La prison avec sursis apparaît ainsi aux yeux des juges comme adéquate pour réguler la fraude fiscale : elle est un moyen de reconnaître le délit, sans trop pénaliser celui qui en est l'auteur.

32 Chiffres clé de l'administration pénitentiaire 2013. http://www.justice.gouv.fr/prison-et-reinsertion10036/les-chiffres-clefs-10041/

${ }^{33}$ Voies et Moyens, Annexe au projet de loi et finances pour 2014, t. I : les recettes.

${ }^{34}$ Loïc WACQUANT, Punishing the Poor: The Neoliberal Government of Social Insecurity, Duke University Press.,2009. 
Les tribunaux britanniques font preuve d'une moindre clémence à l'égard des délinquants fiscaux. Introduites en 2003, les Sentencing guidelines y ont fortement contribué $^{35}$ : elles ont limité le pouvoir discrétionnaire des juges dans le choix de la sentence. Depuis la fin des années 2000, une part importante des fraudeurs fiscaux est ainsi condamnée à de la prison ferme, suivie d'une incarcération effective ${ }^{36}$. Avec l'augmentation des poursuites dirigées contre des fraudes fiscales de faible envergure, la part des peines de prison ferme et leur durée a diminué depuis 2012-2013, mais celles-ci concernent toujours $36 \%$ des délinquants fiscaux ${ }^{37}$. Cette sévérité relative des juridictions outre-Manche se retrouve dans l'usage des peines alternatives et complémentaires.

\section{L'infamie}

Du côté britannique, les fraudeurs qui ne sont pas condamnés à de la prison ferme ne sont pas pour autant dispensés de toute peine effective. Des community orders (travaux d'intérêt général) sont prononcés contre environ $15 \%$ des délinquants ${ }^{38}$. C'est la peine recommandée par les Guidelines lorsque le délinquant a un faible niveau de culpabilité (ce qui recouvre essentiellement une attitude suiviste) et lorsque la fraude met en jeu un montant limité. Le fait de jouir d'un statut social élevé n'est absolument pas incompatible avec une telle condamnation ${ }^{39}$.

Surtout, la procédure pénale est entourée d'une importante publicité destinée à attirer la honte sur la tête du fraudeur. Le Naming and Shaming ne résulte pas directement de la décision judiciaire, mais d'une politique de communication orchestrée par HM Revenue and Customs. Si le secret fiscal interdit de divulguer les résultats des contrôles fiscaux, l'étiquette de «mauvais contribuable », et plus encore celle de délinquant, lève cette interdiction. Les sentences qui frappent les fraudeurs du fisc sont largement diffusées, parfois accompagnées de photos, dans les brochures, rapports et sites de l'administration comme dans la presse généraliste. Les agents du fisc sont incités à collaborer avec les médias, pour lesquels ils rédigent des communiqués de presse «prêts à l'usage ». La publicité qui peut être attendue d'une procédure pénale est d'ailleurs, selon les agents interrogés, un des critères importants

${ }^{35}$ Julian V. ROBERTS, “Sentencing Guidelines and Judicial Discretion. Evolution of the Duty of Courts to Comply in England and Wales", British Journal of Criminology, 51 (6), 2011, pp. 997-1013.

${ }^{36}$ Sentencing Council, Fraud Offences Sentencing Data, June 2013, p. 11.

${ }^{37}$ Freedom of information request.

${ }^{38}$ Sentencing Council, Fraud Offences Sentencing Data, June 2013, p. 11.

${ }^{39}$ Voir par exemple la condamnation de l'agent des stars, Richard Hillgrove, à 200 heures de travaux d'intérêt général par la Bristol Crown Court, 27 mai 2014. 
dans la décision de poursuivre ou non un contribuable. Même quand elles ne se soldent pas par une peine de prison ferme, les poursuites pénales stigmatisent le délinquant.

La situation est très différente en France. Si les juges français écartent presque systématiquement l'emprisonnement ferme, ils utilisent très peu les autres sanctions disponibles (à l'exception de la prison avec sursis). Des travaux d'intérêt général -pour lesquels l'accord du condamné doit être recueilli- ne sont jamais assignés aux délinquants fiscaux. L'idée même d'y recourir paraît complètement incongrue aux magistrats interrogés. A leurs yeux, la vertu de réinsertion d'une telle peine la réserve à un délinquant sans travail et marginalisé ; c'est implicitement admettre qu'à la différence du chauffard ou du fraudeur aux allocations sociales auxquels de tels travaux sont couramment assignés, le délinquant fiscal ne commet pas une incivilité qui le place en marge du contrat social. Les magistrats français privilégient d'ailleurs systématiquement les peines qui ne mettent pas en danger l'intégration sociale du fraudeur fiscal. Ils ne prononcent ainsi que très rarement la privation des droits civiques, civils ou de famille ( $5 \%$ des cas) et, le cas échéant, pour des durées courtes ( 1 ou 2 ans). Ils acceptent assez facilement que la condamnation ne soit pas inscrite sur le casier judiciaire, lorsque le condamné a payé sa dette fiscale et lorsqu'une telle mention lui interdirait d'accéder à un emploi public ou réglementé. Toutes les sanctions qui pourraient imposer au contribuable les stigmates du délit sont écartées. En 2010, les Sages du Conseil constitutionnel ont fait disparaître, pour plusieurs années, la publication systématique des jugements de condamnation pour fraude fiscale, jugée incompatible avec le principe d'individualisation des peines ${ }^{40}$. Si cette peine peut à nouveau être prononcée, elle est peu utilisée en pratique, ou neutralisée par des publications dans des médias à faible audience.

Cette attitude compréhensive des juges français tient sans doute, en partie, à leur proximité sociale avec ces contribuables ${ }^{41}$. Mais les agencements institutionnels ont aussi un rôle dans l'incapacité de la procédure pénale à transformer le fraudeur du fisc en véritable délinquant.

En France, les moyens d'enquête musclés (garde à vue, auditions de témoins...), qui ne peuvent être mis en œuvre qu'après la transmission du dossier fiscal au procureur, sont très peu utilisés à l'encontre des délinquants fiscaux. Dans un contexte de pénurie des ressources en temps et en hommes, les affaires fiscales sont rarement considérées comme prioritaires. D’abord, elles arrivent devant l'institution judiciaire plusieurs années après la réalisation de la

\footnotetext{
4010 décembre 2010, 2010-72/75/82 QPC.

${ }^{41}$ Marshall B. Clinard et Peter C. Yeager, Corporate crime, New York, Free Press, 1980, p. 286-294.
} 
fraude, alors que le contribuable est parfaitement averti de ce qui lui est reproché et a eu tout le temps nécessaire pour dissimuler les preuves. Ensuite, la longue procédure de sélection de ces affaires leur a donné l'apparence de «dossiers bouclés »: si l'administration fiscale fait, en réalité, rarement apparaître les circuits de la fraude ou sa logique économique, les éléments qu'elle met au jour suffisent pour établir l'intention d'éviter l'impôt et obtenir une condamnation. Dans bien des cas, l'enquête judiciaire se résume à une convocation de la personne mise en cause à un entretien; si le suspect accepte de s'y rendre, il devra juste répondre à quelques questions essentiellement destinées à recueillir un aveu mais aucune investigation supplémentaire ne sera entreprise. L'audience ne met pas davantage en scène la qualité de délinquant du prévenu. Celui-ci comparaît libre; il n'est ni encadré par des policiers ni enfermé dans un box, comme c'est le cas devant les Crown Courts britanniques.

La présence du jury dans les affaires de délinquance économique et financière est régulièrement discutée au Royaume-Uni, pour son caractère coûteux et l'imprévisibilité de ses décisions. Mais l'exemple français montre qu'accorder cette compétence à des magistrats professionnels peut également avoir des inconvénients, notamment quand elle s'accompagne du passage à une procédure principalement écrite. Même spécialement formés à l'exercice de la justice, les juges sont souvent des profanes de la fiscalité. Pendant le bref temps de l'audience, ils se focalisent sur les aspects techniques ; en présence de fraudes complexes, les débats portent essentiellement sur la machinerie de l'illégalisme fiscal. La malhonnêteté du contribuable est d'autant plus reléguée au second plan que l'audience ouvre une nouvelle page. Attachés à un idéal d'indépendance judiciaire, les magistrats privilégient leur propre ressenti sur celui de l'inspecteur des impôts qui a mené l'enquête et qui, contrairement à la pratique britannique dans le cadre du procès, n'est jamais appelé à l'audience comme témoin. A défaut de faire complètement disparaître les éléments rassemblés par le fisc, le prévenu qui sait répondre aux attentes des juges ${ }^{42}$, en acquittant sa dette fiscale et en faisant amende honorable, parvient à se défaire de l'image d'un contribuable récalcitrant et arrogant. Il met ainsi le temps de l'audience à profit pour signifier à ses juges qu'il n'a pas les traits d'un escroc habituel ${ }^{43}$ et éviter une peine afflictive.

42 Alexis SPIRE et Katia WEIDENFELD, «Le tribunal administratif : une affaire d'initiés ? Les inégalités d'accès à la justice et la distribution du capital procédural », Droit et Société, 79 (3), 2011, pp. 689713.

\footnotetext{
${ }^{43}$ James William COLEMAN, The criminal elite: Understanding white-collar crime, Macmillan, 2005.
} 
En définitive, les seules condamnations prononcées ayant un effet réel sont celles qui ont une incidence financière : amendes, solidarité du dirigeant à la dette fiscale de l'entreprise ou confiscation.

L'administration fiscale française paraît très bien s'accommoder de cette situation. En France -comme d'ailleurs en Grande-Bretagne ${ }^{44}$, elle place tous ses espoirs dans le développement de la peine de confiscation. Saisir le patrimoine du fraudeur n'est toutefois qu'un moyen, pour le fisc, d'obtenir la restitution, en nature, de ce qui lui a été soustrait.

Officiellement, les hauts-fonctionnaires français chargés de mettre en œuvre la politique pénale, défendent l'idée que la condamnation a une fonction dissuasive ; il s'agit de freiner, par l'exemple, l'accomplissement de comportements indésirables. Mais cette argumentation est très éloignée de ce qui se passe en réalité. Si l'on excepte quelques affaires -concernant principalement des hommes politiques- relayées par le Parquet national financier, l'administration fiscale communique peu sur ses actions répressives. Alors que la répression de la petite délinquance fait l'objet d'un large écho médiatique, la théâtralisation de la lutte contre la fraude fiscale s'est arrêtée à l'enceinte parlementaire. Le rôle d'expression privilégiée des valeurs de la République jouée par la justice pénale ${ }^{45}$ n'est pas mis à profit pour manifester la centralité du respect des obligations fiscales par les citoyens. La procédure pénale est surtout, et peut-être seulement, un instrument supplémentaire pour faire rentrer l'argent dans les caisses de l'Etat. En France, la répression manque ainsi sa fonction originale. Moins que d'imposer les stigmates du délinquant au fraudeur fiscal, il s'agit avant tout de renforcer les pouvoirs des agents du fisc.

De part et d'autre de la Manche, les discours sont à l'unisson : il s'agit d'accompagner le mouvement international d'indignation à l'encontre de l'évasion fiscale par le renforcement de la pénalisation de la fraude fiscale. Cette volonté s'est effectivement traduite, au RoyaumeUni, par une augmentation du nombre des poursuites et, en France, par une diversification de celles-ci pour atteindre des fraudes de plus grande envergure. Ces efforts laissent néanmoins perdurer une gestion différentielle des illégalismes fiscaux qui permet aux plus puissants d'échapper aux poursuites.

Mais la comparaison avec la pratique britannique met surtout en relief la distance existant, en France, entre l'enjeu réel de la pénalisation de la fraude fiscale et l'objectif

\footnotetext{
${ }^{44}$ National Audit Office, Confiscation orders, 17 décembre 2013.

${ }^{45}$ Anne SIMONIN, Le Déshonneur dans la République. Une histoire de l'indignité 1791-1958, Paris, Grasset, 2008, p. 21 sq.
} 
répressif affiché. La rareté des condamnations à des peines afflictives et la faiblesse de la communication publique sur les procédures pénales conduisent à faire de celles-ci principalement un instrument financier destiné à améliorer le recouvrement de l'impôt fraudé. En amont, la menace d'engager des poursuites pour fraude fiscale, qui exige une plainte du ministre du Budget, peut constituer pour les agents du fisc un moyen de pression destiné à obtenir la coopération d'un contribuable. En aval, les seules peines non virtuelles prononcées par les tribunaux français sont celles ayant une fonction pécuniaire.

Les politiques pénales française et britannique revendiquent toutes deux un effet dissuasif, mais celui-ci est appelé à jouer de deux manières très différentes. Au Royaume-Uni, il s'agit de susciter chez les contribuables la crainte d'une sanction afflictive, alors qu'en France, l'objectif principal est de convaincre les contribuables qu'ils n'échapperont pas au remboursement des impôts fraudés. Ce rôle assigné aux poursuites pénales en matière fiscale manifeste bien une conception de l'impôt comme un outil essentiellement financier, reléguant au second plan sa valeur politique de définition d'un territoire et d'expression d'une communauté d'intérêts. 